\title{
Scenario-Based Fault-Tolerant Model Predictive Control for Diesel-Electric Marine Power Plant
}

\author{
Torstein Ingebrigtsen $\mathrm{B} \emptyset$ \\ and Tor Arne Johansen \\ Center for Autonomous Marine Operations and Systems, \\ Department of Engineering Cybernetics, \\ Norwegian University of Science and Technology \\ 7491 Trondheim, Norway \\ Email: torstein.bo@itk.ntnu.no, tor.arne.johansen@itk.ntnu.no
}

\begin{abstract}
Diesel-electric propulsion is often used for vessels with dynamic positioning systems. For such vessels any single point failure should not lead to loss of position. One failure case is that a generator set is suddenly disconnected. This loss of a power producer gives a drop of the power bus frequency. A blackout will occur if the frequency drops too much, it is therefore required to keep the frequency high enough to withstand such a failure. Load reduction is often used to avoid blackouts due to such faults. The power management system will detect that a fault has occurred, and will reduce the load of some consumers to reduce the overload and recover the plant. In this paper, a controller is suggested which explicitly handle the requirements for the failure scenarios. This method is a practical approach to handle the safety requirements, in addition to existing load reduction. The plant can run with fewer connected generator sets by better control of the safety requirements, which will reduce the fuel consumption.
\end{abstract}

Keywords-Fault tolerancy, Automatic generation control, Marine safety, Optimal control.

\section{INTRODUCTION}

Diesel electric propulsion is a system where diesel generator sets (a diesel engine connected to a generator) produce electrical power, which is used by consumers such as thruster drives, drilling drives, and hotel loads. The system is flexible, since generator sets can be started and stopped when the power demand changes.

A Dynamic positioning (DP) system is used for controlling position and heading, this is done by using thrusters to counteract the environmental forces. It is required that any single point failure should not lead to a loss of position, for vessels with DP of class 2 or 3 [1]. This also means that a loss of a generator set should not lead to a blackout.

When a sudden disconnection of generators occurs, the load from the disconnected generator is distributed to the remaining generators connected to the same power bus. The frequency will then decrease due to slow dynamics in the air system of the diesel engine. Rules given by class societies states that the frequency should stay between $90-110 \%$ of rated frequency during transients [2]. Therefore, a safety margin is needed to avoid trip of under-frequency protection relay after a disconnection of a generator. Today this is handled by trying to control the frequency to the rated value. However, the controller will have more freedom for optimization if the frequency could vary between some dynamical safety constraints. It may also be impossible to find static safety constraints which are appropriate for all modes of the system. Therefore, the plant can be safer and operate more optimally by explicitly including the worst-case fault scenarios in the controller.

Diesel engines have typically their maximum efficiency at $70 \%-80 \%$ of their rated power [3]. For some vessels which needs steam are the thrusters set up to use excess power, such that the generator sets can produce enough heat to produce steam [4], this is called thruster biasing. It is reported that around $10 \%$ of the fuel consumption can be reduced if two generator sets are used instead of three [5]. This is due to the need of thruster biasing in low load conditions, which is removed when fewer generator sets are used. This can be achieved by more explicit control of the safety margins.

Model Predictive Control (MPC) is a method where the control input is optimized with respect to a cost function. MPC has gained much interest due to its ability to handle constraints and minimize cost. It has also been shown that MPC has some inherent Fault Tolerant Control (FTC) properties [6]. FTC is used when a controller is required to control the plant before and after a fault. In passive FTC a controller is chosen such that it will work for both the fault-free plant and plants with faults. Active FTC switches between controllers when a fault occurs; a method for detecting and identifying the fault is therefore required [7]. MPC has also been suggested for proactive fault tolerant control of chemical plants [8].

Each of the possible faults of a power plant can be seen as a scenario for future operation of the plant. Therefore, the controller suggested in this paper, simulates multiple scenarios to make sure that the controller can recover the system if one of the faults occurs. Scenarios are already used in a combination with MPC in robust model predictive control $[9,10]$. In finance, scenarios are used for optimization of hedge options [11].

Load reduction is typically used in marine power plants to avoid blackout after loss of power producers [3]. This can be done by reducing the load of some consumers if the frequency decreases under certain levels [5]. Event based load reduction has also been implemented, where the load is reduced if certain events occurs, such as trip of a generator breaker [12]. 


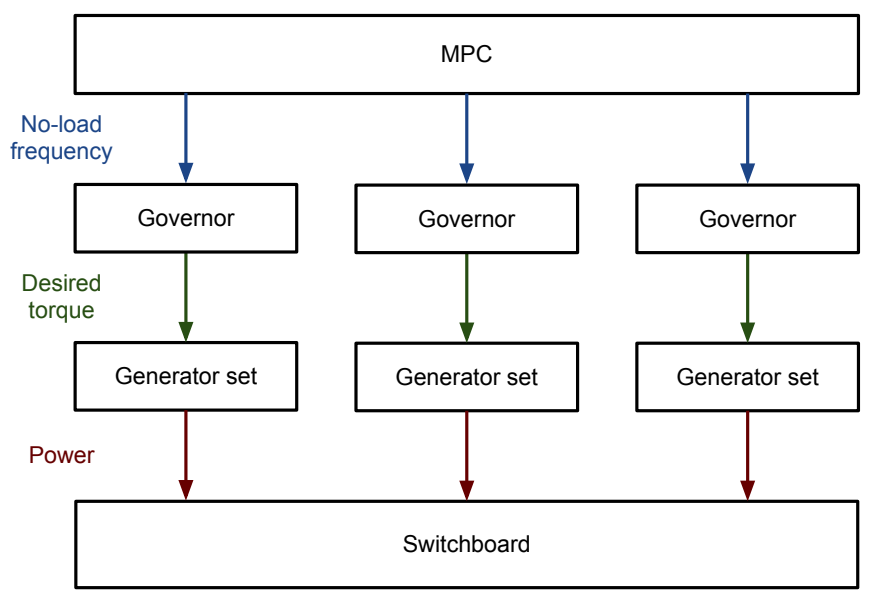

Fig. 1. Control architecture of the power plant. The designed controller (MPC) gives a no-load frequency value to the governor. The governor uses this signal to control the frequency of the generator set, by adjusting the desired torque (fuel command) to the generator set. The generator sets produces power which is fed to the switchboard.

Typically is the reduction done by frequency converters, since they can react within an order of 100 milliseconds. An observer based load reduction system has also been proposed, where the load reduction is based on the estimated torque deviation between the electrical and mechanical torque [13].

The main contribution of this paper is a controller which makes sure that the power plant can withstand some fault scenarios. The controller is implemented using model predictive control and fault scenarios. A set point is given to the governor of the generator sets, in order to adjust its no-load-frequency in droop mode. This set point minimizes the fuel consumption, makes sure that complete combustion is achieved, and that the plant can be recovered if one of the fault scenarios occurs.

\section{PRoblem Statement}

The problem to be looked on consists of two parts. First the controller should control the frequency and the load sharing such that the plant can be recovered if one of the fault scenarios occurs. Secondly, the controller should detect if it is currently able to recover the frequency if one of the fault scenario occurs given the current state. Last, the controller should minimize the fuel consumption, wear, and emissions.

\section{Model of the Marine Power Plant}

A power plant consisting of three equally sized generator sets will be used to illustrate the properties of the controller. The generator sets are equal and rated to $5 \mathrm{MW}$.

The control hierarchy of the plant is shown in Figure 1. The designed controller (MPC) gives a no-load frequency to the governor, the local controller of the generator set. From the no-load frequency, the frequency of the engine and the power out of the generator is the desired torque calculated and given to the generator set. The generator sets uses this desired torque and a mapping between torque and fuel injection to adjust the fuel injection. The generator set produces power which is delivered to the switchboard and redistributed to the consumers.

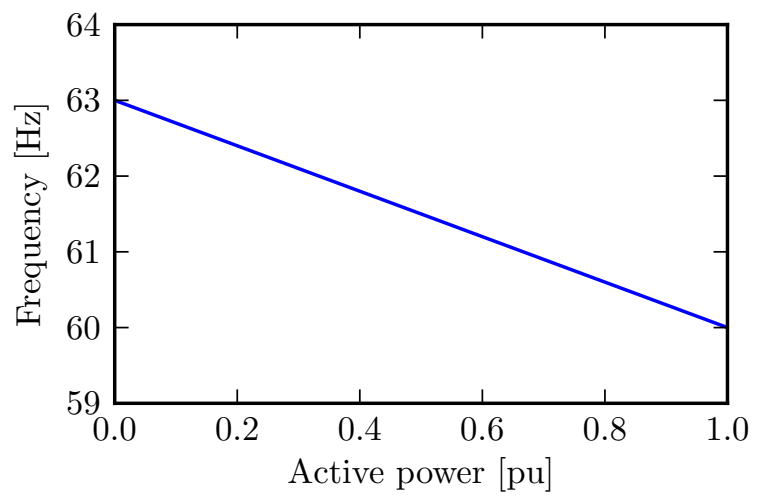

Fig. 2. Droop curve with $5 \%$ Droop and a no-load frequency of $63 \mathrm{~Hz}$.

The desired torque of each diesel engine is controlled by a governor running in droop mode. The reference frequency is found by the droop equation:

$$
\omega_{\text {ref }, i}=\omega_{N L, i}\left(1-\operatorname{Droop} p_{i}\right),
$$

where $\omega_{r e f, i}$ is the reference frequency of Genset $i, \omega_{N L, i}$ is the no-load frequency of Generator $i$, Droop is a positive constant, and $p_{i}$ is the active power out of Generator $i$. A graphical representation of the droop equation is given by the droop curve in Figure 2. This reference frequency is then given to a PID-controller which controls the frequency of the diesel engine to the reference frequency. The no-load frequency is adjusted online by the MPC controller to obtain different load sharing and to change the frequency of the engines.

After a disconnection of one generator the excessive load will be shared among the remaining generators. More fuel must be injected to increase the torque of the remaining engines. However, more air is then needed to achieve complete combustion and it takes some time before the turbocharger has increased the pressure in the inlet manifold. It is therefore important to model the air dynamics, such that the air-to-fuel ratio can be constrained to avoid incomplete combustion. A first order model is therefore used to model the intake air dynamics [14]:

$$
\begin{aligned}
\dot{p}_{i n} & =\frac{1}{T_{i n}}\left(\dot{m}_{f}-p_{i n}\right), \\
\frac{A}{F} & =\frac{\dot{m}_{a}}{\dot{m}_{f}}=\frac{\dot{m}_{a, 0}+\left(1-\dot{m}_{a, 0}\right) p_{i n}}{\dot{m}_{f}}\left(\frac{A}{F}\right)_{N},
\end{aligned}
$$

where $p_{i n}$ is the pressure in the inlet manifold, $T_{i n}$ is the time constant of the inlet manifold pressure, $\dot{m}_{f}$ is the rate of fuel injected in the cylinders, $\frac{A}{F}$ is the air-to-fuel ratio, $\dot{m}_{a}$ is the mass flow of air into the cylinders, $\dot{m}_{a, 0}$ is the air flow during idling of the engine, and $\left(\frac{A}{F}\right)_{N}$ is the air-to-fuel ratio when the engine delivers rated power.

The efficiency of the diesel engine is modeled with Willans approximation [15]. This gives a relationship between the fuel consumption and the delivered power. In Figure 3 is the mapping shown, where a second order polynomial is used for this approximation. 


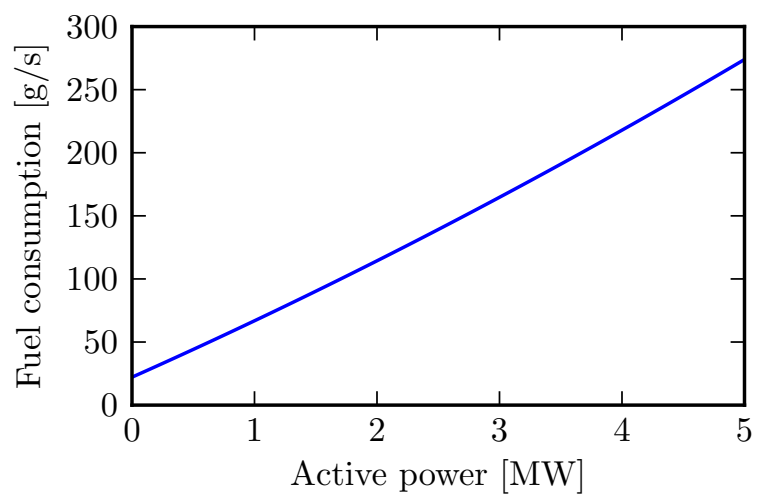

Fig. 3. Fuel consumption of a $5 \mathrm{MW}$ diesel generator set as a function of delivered power.

The dynamics of a generator set can be found by the swing equation and assuming linear damping [16]:

$$
\dot{\omega}_{i}=\frac{1}{2 H_{i}}\left[k_{\dot{m}, i} \dot{m}_{i}-\frac{p_{i}}{\omega_{i}}-D_{f w, i} \omega_{i}\right],
$$

where $\omega_{i}$ is the per-unit electrical frequency of Generator set $i$, $H_{i}$ is the inertia constant of Generator set $i, k_{\dot{m}, i}$ is the gain from fuel rate input to per unit torque, $\dot{m}_{i}$ is the fuel rate, $p_{i}$ is the active power delivered from Generator $i$, and $D_{f w, i}$ is the mechanical damping constant.

When multiple synchronous generators are connected together a strong synchronization torque will keep them running at the same frequency. By using energy balance and assuming that the difference in frequency is small, the equation of the frequency can be found as a function of the total consumed power and the total amount of fuel injected:

$$
\dot{\omega}=\frac{1}{2 H_{T}}\left[\sum_{i} s_{b, i} k_{\dot{m}, i} \dot{m}_{i}-\frac{p_{b u s}}{\omega}-\sum_{i} s_{b, i} D_{f w, i} \omega\right],
$$

where $H_{T}$ the combined inertia constant of all generator sets, $s_{b, i}$ is the ratio between base power of generator set $i$ and total base power, $p_{b u s}$ is the per-unit consumed power,

Note that this equation cannot detect if the frequency of two generator sets oscillates in opposite phase. However, this stability issue is neglected as the governor should take care of it.

\section{Fault TOLERANT MPC}

The idea of the controller is to optimize the frequency and load sharing such that it is able to withstand all of the fault scenarios. This is done by using one prediction internally in the MPC for each fault scenario in addition to a nominal scenario. The nominal scenario is that the plant will stay configured as it is (no faults). The fault scenarios are chosen to be the fault scenarios that we would like to make sure that the plant can recover. These predictions are optimized by adjusting the noload frequency. Each prediction starts with the model given by the nominal scenario for the first time interval, during this interval all predictions have the same no-load frequency. After this time interval the predictions switch to the model for the corresponding scenario, assuming that the fault occurrence will be detected within the first interval.

This means that the controller indirectly finds an optimal frequency which is such that the plant can be recovered if one of the fault scenarios occurs. The controller then tries to control the frequency to the optimal frequency by adjusting the no-load frequency of the governors.

The torque out of the diesel engine will be reduced due to incomplete combustion if the air-to-fuel ratio goes bellow the stoichiometric ratio [15]. The MPC controller will therefore set no-load frequencies such that the governors do not give commands that give too low air-to-fuel ratios. This constraint is given by:

$$
\frac{A}{F} \geq\left(\frac{A}{F}\right)_{\min }
$$

where $\left(\frac{A}{F}\right)_{\min }$ is the minimum allowed air-to-fuel ratio.

A cost function is used to minimize the fuel consumption, this cost function penalize the total consumption of fuel and variations of the frequency. For some initial values it is not possible to find no-load frequencies which makes sure that the frequency is kept within under- and over-frequency limits for all scenarios. This constrain is therefore softened by using slack variables, such that the controller is always able to find a feasible solution to the optimization problem.

\section{Detection of Fault Tolerant Operation}

This method can detect that the plant is not prepared for the fault. The plant cannot recover the simulated faults if the predictions show that the frequency moves out of the allowed range. The minimum and maximum frequency of the predictions can be checked, and additional actions can be taken if they show that the frequency cannot be kept within the allowed range. The action could be to start another generator set or to use other recovery methods such as fast load reduction.

\section{Closed-loop Simulation}

Closed-loop simulations show the performance of the controller. The chosen fault scenarios are that one of each generator is suddenly disconnected. Three fault scenarios are used, such that the disconnection is simulated for each of the generator sets. However, if it is known that one given generator set will disconnect (may be given by alarms or information from protection relays) then only one fault scenario is needed. The load sharing will be changed when only one fault scenario is used, while when one scenario is used per generator set the frequency will be changed.

The load is set to $50 \%$ of the rated power of the connected generator sets and fast load reduction is turned off to clearly illustrate the method. In Figure 4 the closed-loop frequency is presented, with the prediction for frequency of both the nominal and one of the fault scenarios. In Figure 5 is the airto-fuel ratio and no-load frequency for the predictions plotted. Only the prediction for one of the generator sets and one failure scenario is presented. These values are equal for all connected generator sets and all failure scenarios, since the generator sets are equally sized. 


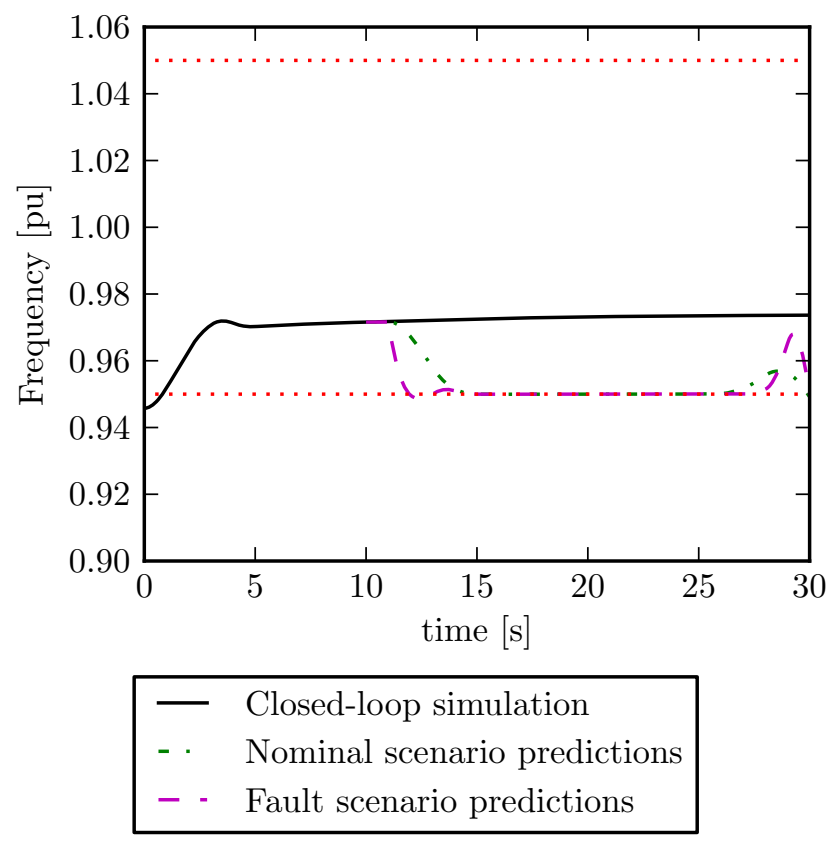

Fig. 4. Closed-loop simulation of marine power plant.

The controller adjust the frequency to a low value, this gives the lowest losses due to the linear damping in the generator set. Note that the prediction of the frequency is equal for both cases during the first time interval of the prediction, and that the closed-loop frequency follows this prediction. Next, the predicted frequency is dropping after this point. For the nominal scenario the frequency drops to reduce the fuel consumption. The frequency of the fault scenarios drops due to the increased load on the remaining generator sets. The no-load frequency of the fault scenario is increased such that more fuel is injected, but still keeping the air-to-fuel ratio high enough to avoid incomplete combustion. The frequency is reduced to the lower limit and a "bump" occurs at the end of all predictions, this is done to reduce fuel consumption of the predictions and is an effect of the controller formulated as an Economic Model Predictive Controller [17]. Notice that this will not occur in practice since only the first interval of the prediction is used, while the rest of the predictions (the tail) are used to make sure that the chosen no-load frequency is safe.

Figure 6 shows the minimum frequency of the predicted scenarios. It is clear from the figure that the plant cannot handle a fault during the first time instant, but the frequency is controlled high enough to withstand the fault scenarios after two or three updates.

\section{CONCLUSION}

This study presents a practical method for implementing dynamical safety constraints of the frequency of diesel-electric power plants. The constraints are based on fault scenarios which are explicitly connected to safety requirements. In this paper a closed loop simulation is presented, with a disconnection of one generator as fault scenario.

A method for detecting that the plant is fault tolerant is

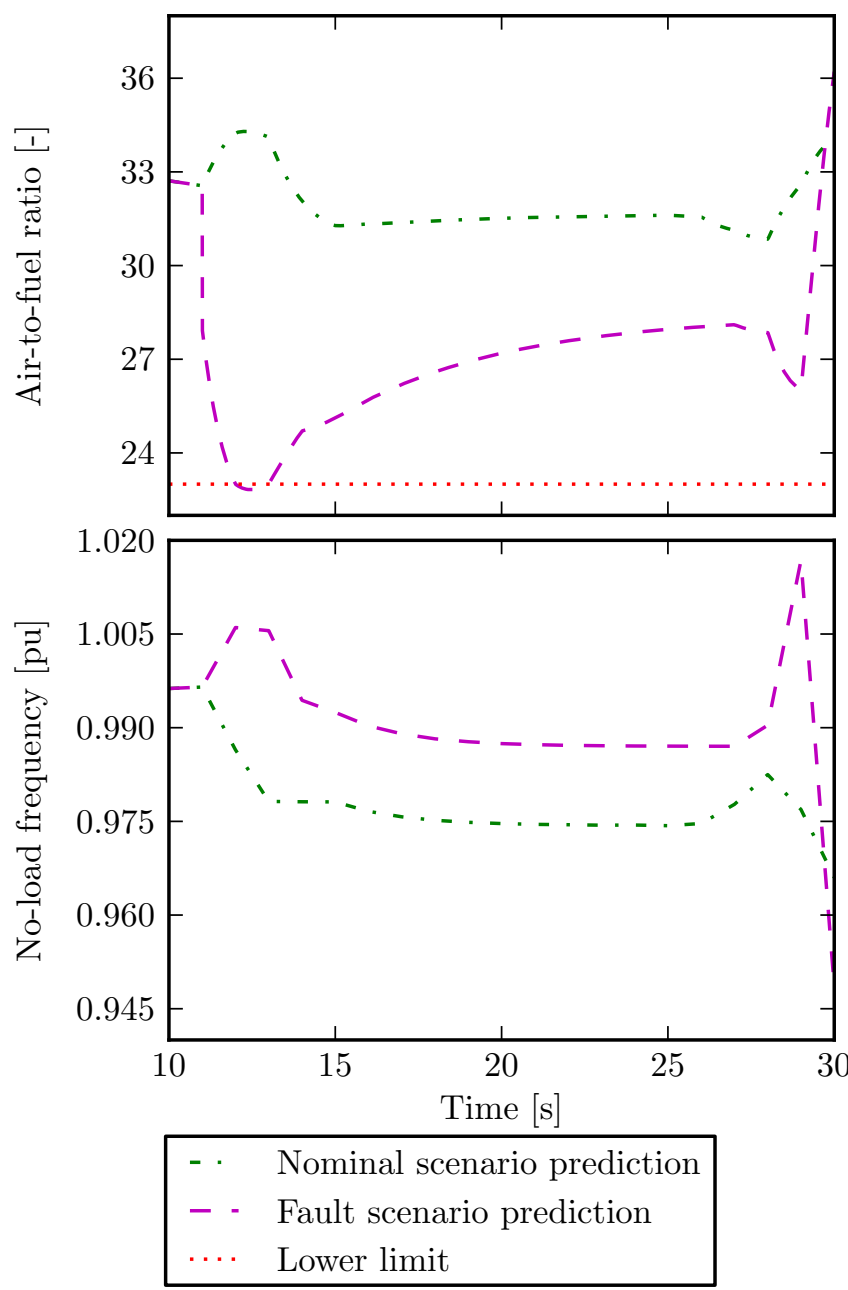

Fig. 5. Prediction of air-to-fuel ratio and no-load frequency for both the nominal and one of the fault scenarios. Note that the air-to-fuel ratio decreases under the lower limit for a short period. This is due to the finite sampling time of the prediction and that the predictions are only constrained at the sampling points.

also presented. This can be used to switch between recovering methods such as with or without load reduction.

\section{APPENDIX \\ LIST OF PARAMETERS}

Time constant of inlet pressure manifold, $T_{i} n \quad 5$ seconds Air flow when idling, $\dot{m}_{a, 0}$ 0.2

Air-to-fuel ratio at rated power $\left(\frac{A}{F}\right)_{N}$ 27

Minimum air to fuel ratio

Inertia constant of generator sets, $H$ 2 seconds Linear damping of generator sets, $D_{f w}$ 0.025

\section{ACKNOWLEDGMENT}

The authors of this paper are funded by the Research Council of Norway, Kongsberg Maritime, and Det Norske Veritas (DNV). 


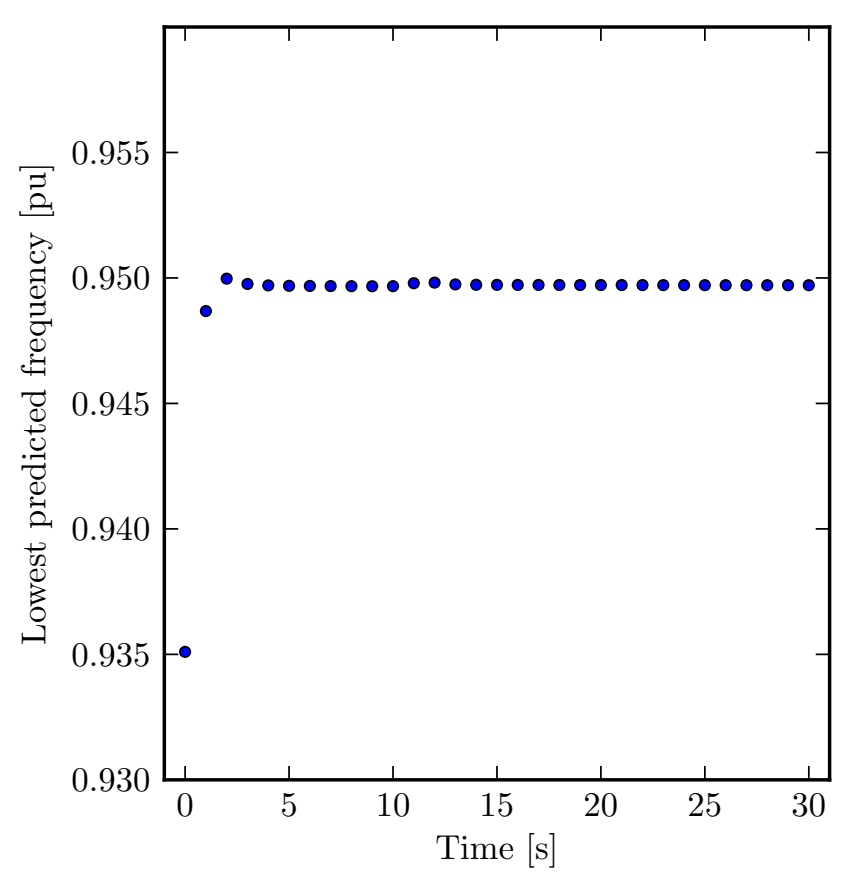

Fig. 6. Calculated lowest frequency of predictions.

\section{REFERENCES}

[1] International Maritime Organization, Guidelines for Vessels with Dynamic Positioning Systems. June 1994. Maritime Safety Committee (MSC) Circular 645.

[2] "Rules for classification of ships," April 2012. Det Norske Veritas, April 2012.

[3] A. K. Ånanes, Maritime Electrical Installations and Diesel Electric Propulsion. 2003.

[4] B. Realfsen, "Reducing NOx Emission in DP2 and DP3 Operations," in Dynamic Positionong Conference, 2009.

[5] J. J. May, "Improving engine utilization on DP drilling vessels," in Dynamic Positioning Conference, 2003.

[6] J. Maciejowski, "Modelling and predictive control: enabling technologies for reconfiguration," Annual Reviews in Control, vol. 23, pp. 13-23, 1999.

[7] M. Blanke, M. Kinnaert, J. Lunze, and M. Staroswiecki, "Diagnosis and Fault-Tolerant Control," in Diagnosis and Fault-Tolerant Control, Springer Berlin Heidelberg, 2nd ed., 2006.

[8] L. Lao, M. Ellis, and P. D. Christofides, "Proactive Fault-Tolerant Model Predictive Control," AIChE Journal, 2013.

[9] G. C. Calafiore, S. Member, and L. Fagiano, "Robust Model Predictive Control via Scenario Optimization," IEEE Transactions on Automatic Control, vol. 58, no. 1, pp. 219-224, 2013.

[10] D. Bernardini and A. Bemporad, "Scenario-based model predictive control of stochastic constrained linear systems," Proceedings of the 48h IEEE Conference on Decision and Control (CDC) held jointly with 2009 28th Chinese Control Conference, pp. 6333-6338, Dec. 2009.

[11] A. Bemporad, L. Bellucci, and T. Gabbriellini, "Dynamic option hedging via stochastic model predictive control based on scenario simulation," Quantitative Finance, pp. 1-13, Feb. 2012.
[12] T. Lauvdal and A. K. Ådnanes, "Power management system with fast acting load reduction for DP vessels," in Dynamic Positioning Conference, 2000.

[13] D. Radan, Integrated control of marine electrical power systems. Doctoral thesis, monograph, Norwegian University of Science and Technology, 2008.

[14] A. Veksler, T. A. Johansen, and R. Skjetne, "Transient power control in dynamic positioning-governor feedforward and dynamic thrust allocation," IFAC Conference on Manoeuvring and Control of Marine Craft, Arenzano, 2012.

[15] L. Guzzella and C. H. Onder, Introduction to Modeling and Control of Internal Combustion Engine Systems. Berlin, Heidelberg: Springer Berlin Heidelberg, second ed., 2010.

[16] R. Skjetne, "Modeling a diesel-generator power plant." Lecture notes, Norwegian University of Science and Technology, 2010.

[17] L. Grüne, "Economic receding horizon control without terminal constraints," Automatica, vol. 49, no. 3, pp. 725734, 2013. 